\title{
Tautomeric Conflicts in Forty Small-Molecule Databases
}

\author{
Devendra K. Dhaked ${ }^{1}$, Marc C. Nicklaus ${ }^{1 *}$ \\ ${ }^{1}$ Computer-Aided Drug Design Group, Chemical Biology Laboratory, Center for Cancer \\ Research, National Cancer Institute, NIH, Frederick, MD 21702, USA.
}

\begin{abstract}
We have analyzed forty different databases ranging in size from a few thousand to nearly 100 million molecules, comprising a total of over 200 million structures, for their tautomeric conflicts. A tautomeric conflict is defined as an occurrence of two or more structures within a data set identified by the tautomeric rules applied as being tautomers of each other. We tested a total of 119 detailed tautomeric transform rules expressed as SMIRKS, out of which 79 yielded at least one conflict. The databases analyzed spanned a wide variety of types including large aggregating databases, drug collections, and experimentally based structure collections. Almost all databases analyzed showed intra-database tautomeric conflicts. The conflict rates as percentage of the database were typically in the few tenths of a percent range, which for the largest databases amounts to $>100,000$ cases per database.
\end{abstract}

\section{INTRODUCTION}

Tautomerism is a ubiquitous phenomenon in chemistry. Estimates of what percentage of structures in a small-molecule database are capable of some kind of tautomerism have typically been above $10 \% \%^{1,2}$ and if analyzed by comprehensive chemoinformatics rules, - were found to be as high as $66 \%$ of the cases. ${ }^{3}$ Our recent study using $80+$ tautomeric rules indicated the presence of an average of $71 \%$ tautomerism-capable molecules across 9 databases from a collection of $>400$ million structures. ${ }^{4}$ It is therefore no surprise that existing databases may show cases where molecules are listed as separate record in it that rule-based approaches define as tautomers of each other. We have termed such cases "tautomer conflicts."

We previously analyzed over 150 databases, totaling more than 103 million structures, for the tautomeric conflicts in each database as well as across all databases combined. ${ }^{3}$ We also analyzed a medium-size database ( $\sim 6$ million records) for its tautomeric conflicts by both chemoinformatics and experimental analysis, which computationally identified more than 31,000 conflicts, out of which more than 100 were experimentally tested. ${ }^{5}$ This showed that in most cases, the chemoinformatics rules had correctly predicted the analytical identity of the tautomeric tuple members. 
For this study, we significantly broadened both the number of tautomeric transform rules applied, ${ }^{4}$ as well as the total number of compounds studied.

We need to strongly emphasize that finding a tautomer conflict in a database is not identical with having identified a problem in the database. There may be many reasons why tautomers of each other may be present in different database records. A tautomer conflict as identified in this study should therefore rather be seen as an alert to perhaps take a closer look at tautomer tuples to investigate if they truly create an issue in the database.

\section{DATA AND METHODS}

\section{$\underline{\text { Databases }}$}

We downloaded 40 databases, ranging in size from nearly 100 million to a few thousand structures (Table 1). The total number of (non-unique) structures analyzed is more than 210 million. 
Table 1. Databases used in this study.

\begin{tabular}{|c|c|c|c|c|}
\hline Database & Type & $\begin{array}{l}\text { Molecule } \\
\text { count in } \\
\text { database }\end{array}$ & $\begin{array}{l}\text { Date } \\
\text { accessed }\end{array}$ & $\begin{array}{l}\text { Way of access } \\
<\text { download URL; or other way of getting it> }\end{array}$ \\
\hline PubChem & A & $96,502,282$ & Oct 2018 & https://pubchem.ncbi.nlm.nih.gov/ \\
\hline eMolecules & $\mathrm{B}, \mathrm{S}, \mathrm{A}$ & $23,217,671$ & Nov 2019 & https://www.emolecules.com/info/plus/download-database \\
\hline $\begin{array}{l}\text { SureChEMBL } \\
\text { (Patents) }\end{array}$ & $\mathrm{P}$ & $19,334,472$ & Dec 2019 & https://www.surechembl.org/search/ \\
\hline MCULE & $\mathrm{B}, \mathrm{O}$ & $18,445,974$ & Nov 2019 & https://mcule.com/database/ \\
\hline Ambinter & $\mathrm{S}$ & $9,837,722$ & Nov 2019 & http://www.ambinter.com/ \\
\hline $\begin{array}{l}\text { AMS Screening } \\
(2019 \text { Q4) }\end{array}$ & $\mathrm{S}$ & $8,122,497$ & Dec 2019 & Obtained by subscription to ChemNavigator databases \\
\hline MolPort & $\mathrm{B}, \mathrm{S}$ & $7,615,837$ & Nov 2019 & https://www.molport.com/ \\
\hline Enamine & $\mathrm{B}, \mathrm{S}, \mathrm{O}$ & $3,856,359$ & Nov 2019 & https://enamine.net/ \\
\hline InterChim & S & $2,881,727$ & June 2019 & http://www.interchim.com/ \\
\hline AsisChem & S & $2,107,628$ & Dec 2019 & https://asischem.com/ \\
\hline ChEMBL & $\mathrm{L}$ & $1,820,035$ & Dec 2019 & https://www.ebi.ac.uk/chembl/ \\
\hline $\begin{array}{l}\text { Princeton } \\
\text { Biomolecular } \\
\text { Research }\end{array}$ & $\mathrm{B}, \mathrm{S}$ & $1,780,907$ & Nov 2019 & http://www.princetonbio.com/ \\
\hline Life Chemicals & $\mathrm{B}, \mathrm{F}, \mathrm{O}$ & $1,445,268$ & Nov 2019 & https://lifechemicals.com/ \\
\hline ChemDiv & $\mathrm{B}, \mathrm{O}$ & $1,444,422$ & Nov 2019 & https://www.chemdiv.com/ \\
\hline
\end{tabular}




\begin{tabular}{|c|c|c|c|c|}
\hline $\begin{array}{l}\text { Vitas-M } \\
\text { Laboratory }\end{array}$ & $\mathrm{B}, \mathrm{S}$ & $1,441,235$ & Nov 2019 & https://vitasmlab.biz/ \\
\hline Chemspace & $\mathrm{B}, \mathrm{S}, \mathrm{F}$ & $1,381,383$ & Nov 2019 & https://chem-space.com/ \\
\hline ChemBridge & $\mathrm{S}$ & $1,243,316$ & June 2019 & https://www.chembridge.com/ \\
\hline Innovapharm & $\mathrm{B}, \mathrm{S}, \mathrm{F}, \mathrm{O}$ & $1,123,797$ & Dec 2019 & https://innovapharm.com.ua/ \\
\hline TimTec & $\mathrm{B}, \mathrm{S}, \mathrm{O}$ & $1,086,513$ & Nov 2019 & $\begin{array}{l}\text { https://www.timtec.net/ and } \\
\text { https://www.timtec.net/home/download-databases.html }\end{array}$ \\
\hline Alinda & $\mathrm{B}, \mathrm{S}$ & 929,626 & Dec 2019 & http://www.alinda.ru/synthes_en.html \\
\hline US EPA & $\mathrm{R}$ & 848,945 & Dec 2019 & https://comptox.epa.gov/dashboard/downloads \\
\hline UORSY & $\mathrm{S}, \mathrm{F}, \mathrm{O}$ & 681,161 & Dec 2019 & https://uorsy.com/ \\
\hline Asinex & $\mathrm{B}, \mathrm{S}, \mathrm{F}$ & 642,206 & Nov 2019 & http://www.asinex.com/ \\
\hline InterBioScreen & $\mathrm{B}, \mathrm{S}, \mathrm{F}, \mathrm{O}$ & 572,055 & Nov 2019 & https://www.ibscreen.com/ \\
\hline Otava & $\mathrm{B}, \mathrm{S}, \mathrm{F}, \mathrm{O}$ & 566,491 & Dec 2019 & https://www.otavachemicals.com/ \\
\hline CSD Organic & $\mathrm{E}$ & 319,204 & Dec 2018 & https://www.ccdc.cam.ac.uk/ \\
\hline HTS Biochemie & $\mathrm{B}, \mathrm{S}, \mathrm{O}$ & 290,883 & Nov 2019 & http://www.hts-biochemie.de/hts-en/index.php \\
\hline ChemBank & $\mathrm{E}$ & $1,530,003$ & Jan 2020 & $\begin{array}{l}\text { https://data.broadinstitute.org/chembank/assay (Full set of } \\
\text { molecules privately shared with us }{ }^{6} \text { ) }\end{array}$ \\
\hline Key Organic & $\mathrm{B}, \mathrm{S}, \mathrm{F}, \mathrm{O}$ & 202,241 & Nov 2019 & https://www.keyorganics.net/downloads-bionet-databases/ \\
\hline ChemBlock & $\mathrm{B}, \mathrm{S}$ & 131,662 & June 2019 & http://www.chemical-block.com/ \\
\hline Maybridge & $\mathrm{B}, \mathrm{S}, \mathrm{F}$ & 126,414 & Nov 2019 & ps://www.maybridge.com/portal/alias_Rainbow/lang_en/tabID_177/Deskto \\
\hline
\end{tabular}




\begin{tabular}{|c|c|c|c|c|}
\hline HMDB & $\mathrm{M}$ & 113,983 & Nov 2019 & http://www.hmdb.ca/downloads \\
\hline ChEBI & A & 103,104 & Nov 2019 & https://www.ebi.ac.uk/chebi/downloadsForward.do \\
\hline FDA DailyMed & $\mathrm{R}$ & 60,095 & Feb 2020 & $\begin{array}{l}\text { ftp://public.nlm.nih.gov/nlmdata/.dailymed/substance_indexing } \\
\text { spl_files.zip }\end{array}$ \\
\hline PDB ligand & $\mathrm{E}$ & 29,877 & Aug 2019 & http://ligand-expo.rcsb.org/ld-download.html \\
\hline Combiphos & & & & \\
\hline Catalyst & $\mathrm{O}$ & 19,583 & Dec 2019 & https://www.combiphos.com/products \\
\hline MolMall-MDPI & $\mathrm{O}$ & 15,309 & Dec 2019 & http://www.molmall.net/download.html \\
\hline Cayman & $\mathrm{O}$ & 13,860 & Nov 2019 & https://www.caymanchem.com/ \\
\hline DrugBank (5.1.4) & $\mathrm{D}$ & 10,632 & Aug 2019 & https://www.drugbank.ca/releases/5-1-4 \\
\hline DrugCentral & $\mathrm{D}$ & 4,531 & Dec 2019 & http://drugcentral.org/download \\
\hline
\end{tabular}

Note: "A": Aggregating database; "S": Screening sample supplier; "L": Literature extractions; "R": Regulatory agency database; "E": Experimental results database; "D": Drug database; "M": Metabolite. "B": Building blocks; "F": Fragment or scaffold library; "P": Patent; "O": Other. 
This set spans a wide variety of types of databases: large aggregated databases (e.g., PubChem); screening sample (and other commercially available) catalogs (e.g., Ambinter); databases of regulatory agencies (e.g., US EPA); experimental results (e.g., CSD Organic); drug databases (e.g., DrugBank); human metabolite (e. g. HMDB); patent (e.g. SureChEMBL) and others.

\section{Algorithmic Approaches and Software Used}

The analyses of tautomeric conflicts were performed with the chemoinformatics toolkit CACTVS. ${ }^{7}$ Version 3.4.8.12 of CACTVS was used. CACTVS allows the user to calculate a number of identifiers (hashcodes) that are sensitive to different chemical features such as formal charges in the input structure, presence of isotopically labeled atoms, stereochemistry, etc. One of these features is tautomerism, i.e. if tautomerism invariance is turned on, the identifier returned by CACTVS is the same for all possible tautomers that can be enumerated based on the tautomeric rule set active at the time of execution, otherwise different tautomers receive different identifiers. One such tautomer-invariant identifier is called E_TAUTO_HASH128 (the "E_" standing for: Ensemble property, "ensemble" being the CACTVS term for a compound structure) with 128 bit length (the default hashcode length is 64 bit). Other related hashcodes we computed (named with the terminology "ISOTOPE": sensitive to isotopes; "STEREO": sentive to stereochemistry; "TAUTO": invariant to tautomers) were: tautomer-invariant hashcodes E_ISOTOPE_TAUTO_HASH128, E_STEREO_TAUTO_HASH128, E_ISOTOPE_STEREO_TAUTO_HASH128; and tautomer-sensitive hashcodes E_HASH128, E_ISOTOPE_HASH128, E_STEREO_HASH128, and E_ISOTOPE_STEREO_HASH128.

\section{Tautomerism Rules Used}

We started with 86 previously defined rules ${ }^{4}$ encompassing a wide variety of prototropic, ringchain, and valence tautomeric transforms. They have been thoroughly analyzed as to their prevalence in a variety of databases (including some used in this paper), defined of how many structures in a database are amenable to each rule, i.e. can have more than one tautomer based on this rule; as to their comparison with current InChI (which has some but significantly incomplete handling of tautomerism in its current version, 1.05); and as to other properties. We refer the reader to that analysis ${ }^{4}$ for these detailed analyses. To these 86 SMIRKS, we added 33 SMIRKS from 11 types of ring $\rightleftharpoons$ chain rules ${ }^{8}$ (encoded in a total of 38 SMIRKS). Five of these ring-chain SMIRKS were already part of 86 SMIRKS of our previous study, ${ }^{4}$ which led to a total of 119 SMIRKS used in this study. The entire list of transforms is included as Supplementary Information Table S1. We note that this set can be subdivided into "common" and "rare" rules, simply based on the prevalence observed, with common rules matching millions of structures ${ }^{4}$ in a large database such as PubChem. Fewer than 20 rules are common in this sense. Since a tautomeric conflict requires at least two structures in a database that can interconvert according to our rules but have been labeled as different records by the database provider, i.e. is essentially 
a square function of the occurrence rate, it is obvious that common rules are much more likely to yield tautomeric conflicts than rare ones.

\section{$\underline{\text { Definition and Determination of Tautomeric Conflicts }}$}

We define a tautomeric conflict as the occurrence, within a database, of two or more records labeled by the database provider as structurally different entries, whereas our tautomeric rules indicate that these structures are tautomers of each other. We determine such conflicts by searching for compounds in the database that have the same tautomer-invariant but different tautomer-sensitive hashcodes. Specifically, we used E_ISOTOPE_STEREO_HASH128 and E_ISOTOPE_STEREO_TAUTO_HASH128 to search for tautomeric conflicts. It is important to use stereo-sensitive hashcodes. Non-stereo-sensitive hashcodes ignore stereochemistry anywhere in the molecule. Tautomerism may make some stereogenic centers non-persistent (see below) but many other stereocenters are not affected by tautomerism. Non-stereo-sensitive hashcodes would therefore equate stereoisomers which each other that truly are not tautomers of each other. At the same time, and perhaps somewhat counterintuitively, non-stereo-sensitive hashcodes typically lead to lower conflict counts because they have already projected different stereoisomers onto each other that may be found to be non-persistent based on our rules and thus may receive the same tautomer-invariant hashcodes, while they are often listed as different records in databases.

\section{Determining Tautomeric Rules for Conflicts}

We determined the single transform or sequence of transforms connecting the tuple members with each other, employing an approach bases on tautomeric network as published before ${ }^{5}$, however here using all 119 rules, not just the 20 standard (prototropic) rules of CACTVS. Briefly, we first enumerate all possible tautomers from each tautomeric tuple; then, we generate a tautomer network among those enumerated tautomers. In such a network, one typically finds several pathways that connect one tautomer to the other by different tautomeric transforms. Finally, we search for the shortest pathway, defined by the smallest number of transformation steps within the tautomeric pair.

\section{RESULTS}

\section{$\underline{\text { Intra-Database Conflict Counts }}$}

Table 2 shows the conflict counts for the databases analyzed. While the majority of conflicts stem from tautomer pairs, we also looked for, and found numerous cases of higher-order tuples. We checked for up to 25-tuples. The larger the database, the higher the chance for higher-order tuples. It is therefore no surprise that PubChem leads the pack with two cases of a 25-tuple. The 
total number of intra-database conflicts based on a merger of all 40 databases was $5,401,803$. Table 2 also shows the conflict rate, defined as the number of conflicts divided by the number of molecules in the database (as shown in Table 1). We note that the number of database records involved in the conflicts is at least twice as high since the lowest-order tautomer tuple is a pair. The conflict rates typically fall in the range between $0.1 \%$ and $1 \%$, with the extrema being $11.59 \%$ at the high end and $0.0 \%$ at the low end, and a median of $0.23 \%$. The average conflict rate was about $0.77 \%$. If we remove the outlier of $11.59 \%$ for ChemBank, the median and the average reduce to $0.21 \%$ and $0.48 \%$, respectively. This means that between half and one percent of a database is typically involved in tautomer conflicts. We note that this number is close to the result of our 2010 study, ${ }^{3}$ which had yielded an overall tautomeric conflict rate of $0.3 \%$. We found no conflicts for two databases: Uorsy and Combiphos Catalyst. 
Table 2. Tautomeric conflict counts within each database (intra-database conflicts).

Tautomer conflict tuple size

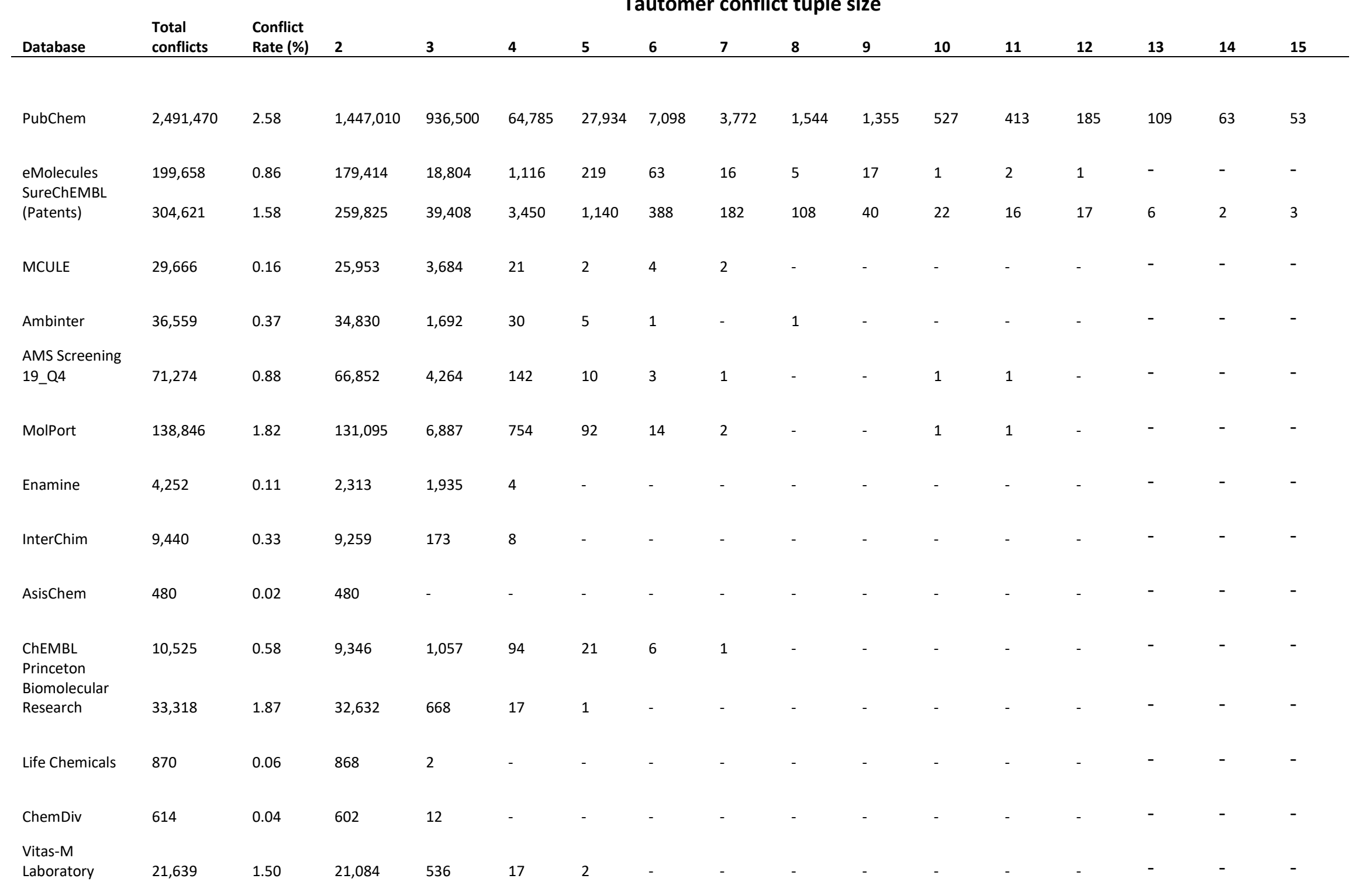




\begin{tabular}{|c|c|c|c|c|c|}
\hline Chemspace & 2,926 & 0.21 & 2,403 & 503 & 16 \\
\hline ChemBridge & 388 & 0.03 & 386 & 2 & - \\
\hline Innovapharm & 3,716 & 0.33 & 3,611 & 101 & 4 \\
\hline TimTec & 1,092 & 0.10 & 1,074 & 18 & - \\
\hline Alinda & 488 & 0.05 & 484 & 4 & - \\
\hline US EPA & 2,777 & 0.33 & 2,478 & 251 & 38 \\
\hline UORSY & 125 & 0.02 & 125 & - & - \\
\hline Asinex & 194 & 0.03 & 174 & 20 & - \\
\hline InterBioScreen & 275 & 0.05 & 272 & 3 & - \\
\hline Otava & 336 & 0.06 & 315 & 17 & 4 \\
\hline CSD Organic & 452 & 0.14 & 443 & 8 & - \\
\hline HTS Biochemie & 324 & 0.11 & 324 & - & - \\
\hline ChemBank & 33,527 & 11.59 & 33,051 & 429 & 39 \\
\hline Key Organic & 309 & 0.15 & 248 & 60 & - \\
\hline ChemBlock & 139 & 0.11 & 139 & - & - \\
\hline Maybridge & 54 & 0.04 & 53 & 1 & - \\
\hline HMDB & 284 & 0.25 & 246 & 33 & 4 \\
\hline
\end{tabular}




\begin{tabular}{|c|c|c|c|c|c|c|c|}
\hline ChEBI & 1,385 & 1.34 & 1,103 & 215 & 33 & 13 & 8 \\
\hline FDA DailyMed & 556 & 0.93 & 513 & 24 & 9 & 4 & 3 \\
\hline PDB ligand & 167 & 0.56 & 148 & 13 & 4 & - & 1 \\
\hline \multicolumn{8}{|l|}{ Combiphos } \\
\hline Catalyst & 4 & 0.02 & 4 & - & - & - & - \\
\hline MolMall-MDPI & 62 & 0.40 & 59 & 2 & 1 & - & - \\
\hline Cayman & 61 & 0.44 & 49 & 10 & 2 & - & - \\
\hline $\begin{array}{l}\text { DrugBank } \\
\text { (5.1.4) }\end{array}$ & 43 & 0.40 & 38 & 3 & 1 & 1 & - \\
\hline DrugCentral & 9 & 0.20 & 9 & - & - & - & - \\
\hline
\end{tabular}

We tested tuple sizes up to 25 but show tuple sizes only up to 15 to limit the size of the table. The entire table with all tuple sizes is provided as Supporting Information Table S2. 


\section{$\underline{\text { Inter-Database Conflicts }}$}

We determined inter-database conflicts by checking, for each tautomeric molecule of a database, for the presence of alternative tautomer(s) in all other 39 databases. We note that these

alternative tautomers may be fully or partially the same across these 39 databases but they had to be different from the query tautomer of the tested database. Table 3 shows the inter-database conflicts, again broken down by tuple sizes similar to Table 2. It is not surprising that the number of inter-databases conflicts is generally higher than that of the intra-databases conflicts (e.g. for PubChem $>5$ million for the former vs. about 1.7 million for the latter) given that structure normalization is most likely different between databases. We also calculated the number of conflicts across all 40 databases combined. Among these 201 million non-unique structures, we found 5,401,803 conflicts. 
Table 3. Tautomeric conflict counts for each database relative to all other databases (inter-database conflicts).

\begin{tabular}{|c|c|c|c|c|c|c|c|c|c|c|c|c|c|c|c|}
\hline \multirow[b]{2}{*}{ Database } & \multicolumn{15}{|c|}{ Inter-database tautomer conflict tuple sizes } \\
\hline & 1 & 2 & 3 & 4 & 5 & 6 & 7 & 8 & 9 & 10 & 11 & 12 & 13 & 14 & 15 \\
\hline PubChem & $5,135,589$ & 257,072 & 18,108 & 4,573 & 1,749 & 1,141 & 543 & 283 & 398 & 91 & 106 & 61 & 116 & 13 & 21 \\
\hline eMolecules & 843,642 & 580,741 & 13,679 & 19,775 & 1,766 & 1,523 & 628 & 1,109 & 150 & 120 & 77 & 68 & 26 & 18 & 8 \\
\hline (Patents) & 698,216 & 134,812 & 12,056 & 5,545 & 1,440 & 1,030 & 534 & 398 & 231 & 102 & 120 & 75 & 82 & 24 & 18 \\
\hline MCULE & $2,566,895$ & 151,734 & 85,015 & 5,680 & 2,463 & 739 & 314 & 126 & 90 & 34 & 18 & 4 & 13 & 3 & - \\
\hline $\begin{array}{l}\text { Ambinter } \\
\text { AMS Screening }\end{array}$ & $1,057,664$ & 546,596 & 16,194 & 17,823 & 2,511 & 1,964 & 680 & 1,290 & 218 & 240 & 101 & 59 & 33 & 42 & 18 \\
\hline (2019 Q4) & $1,391,234$ & 538,174 & 13,708 & 18,622 & 1,719 & 1,548 & 687 & 1,057 & 191 & 158 & 69 & 58 & 29 & 28 & 14 \\
\hline MolPort & 851,328 & 469,692 & 10,831 & 17,277 & 1,566 & 1,382 & 532 & 1,039 & 106 & 93 & 60 & 54 & 19 & 12 & 4 \\
\hline Enamine & 214,666 & 25,102 & 14,707 & 1,252 & 522 & 74 & 65 & 17 & 14 & 4 & 4 & - & 4 & - & - \\
\hline InterChim & 214,882 & 156,473 & 4,189 & 12,018 & 619 & 891 & 443 & 1,328 & 60 & 64 & 61 & 57 & 12 & 9 & 1 \\
\hline AsisChem & 255,351 & 37,918 & 9,908 & 2,205 & 350 & 90 & 18 & 9 & 6 & 2 & 2 & 1 & - & - & - \\
\hline $\begin{array}{l}\text { ChEMBL } \\
\text { Princeton }\end{array}$ & 286,958 & 18,199 & 14,197 & 2,310 & 679 & 357 & 204 & 139 & 73 & 38 & 20 & 7 & 16 & 3 & 4 \\
\hline $\begin{array}{l}\text { Biomolecular } \\
\text { Research }\end{array}$ & 548,344 & 30,672 & 43,184 & 3,457 & 1,317 & 441 & 262 & 104 & 48 & 25 & 10 & 3 & 4 & - & - \\
\hline Life Chemicals & 44,273 & 2,493 & 2,880 & 311 & 82 & 26 & 48 & 19 & 4 & 3 & 1 & - & 1 & - & - \\
\hline $\begin{array}{l}\text { ChemDiv } \\
\text { Vitas-M }\end{array}$ & 121,076 & 9,033 & 13,053 & 1,360 & 366 & 158 & 78 & 31 & 15 & 5 & 3 & - & - & - & - \\
\hline Laboratory & 685,190 & 35,014 & 42,302 & 3,172 & 1,291 & 408 & 196 & 95 & 41 & 22 & 10 & 3 & 3 & - & - \\
\hline Chemspace & 48,927 & 4,246 & 2,047 & 281 & 74 & 29 & 20 & 7 & 8 & 2 & 3 & - & - & - & - \\
\hline ChemBridge & 103,393 & 83,338 & 1,200 & 2,873 & 128 & 156 & 55 & 320 & 24 & 21 & 14 & 7 & 4 & 2 & - \\
\hline Innovapharm & 311,573 & 42,806 & 19,657 & 1,427 & 232 & 95 & 145 & 32 & 15 & 5 & - & - & 2 & - & - \\
\hline TimTec & 379,678 & 20,845 & 25,251 & 2,341 & 691 & 221 & 134 & 63 & 30 & 10 & 4 & 1 & 3 & - & - \\
\hline Alinda & 193,984 & 7,646 & 11,492 & 780 & 252 & 71 & 47 & 16 & 8 & 2 & 2 & - & - & - & - \\
\hline US EPA & 135,800 & 9,458 & 3,017 & 619 & 194 & 146 & 44 & 29 & 21 & 14 & 17 & 11 & 9 & 5 & 1 \\
\hline
\end{tabular}




\begin{tabular}{|c|c|c|c|c|c|c|c|c|c|c|c|c|c|c|}
\hline UORSY & 79,804 & 4,300 & 4,252 & 459 & 86 & 19 & 61 & 11 & 3 & 3 & 1 & - & 2 & - \\
\hline Asinex & 140,134 & 7,192 & 14,932 & 844 & 208 & 54 & 47 & 22 & 9 & 7 & 2 & - & - & - \\
\hline InterBioScreen & 298,326 & 17,003 & 17,766 & 1,460 & 386 & 149 & 122 & 65 & 20 & 11 & 2 & 1 & - & 1 \\
\hline Otava & 109,420 & 9,777 & 10,017 & 934 & 74 & 77 & 72 & 19 & 6 & 4 & - & 1 & 1 & - \\
\hline $\begin{array}{l}\text { CSD Organic } \\
\text { HTS }\end{array}$ & 5,671 & 1,105 & 123 & 56 & 13 & 5 & 5 & 2 & - & - & - & 1 & 1 & 1 \\
\hline Biochemie & 2,999 & 1,604 & 7 & 6 & 2 & - & - & - & - & 1 & - & - & - & - \\
\hline ChemBank & 233,927 & 20,239 & 14,644 & 1,896 & 517 & 241 & 127 & 74 & 39 & 17 & 9 & 1 & 7 & 4 \\
\hline Key Organic & 32,194 & 8,184 & 387 & 297 & 42 & 43 & 18 & 16 & 6 & 2 & 1 & 2 & 1 & - \\
\hline ChemBlock & 23,469 & 2,043 & 3,905 & 426 & 150 & 59 & 22 & 9 & 5 & - & 1 & - & - & - \\
\hline Maybridge & 22,287 & 1,883 & 1,413 & 90 & 29 & 10 & 1 & 1 & - & 1 & 1 & - & - & - \\
\hline HMDB & 8,353 & 822 & 272 & 74 & 54 & 25 & 14 & 5 & 9 & 8 & 3 & 1 & 1 & 4 \\
\hline ChEBI & 31,223 & 3,104 & 781 & 291 & 165 & 80 & 28 & 25 & 23 & 17 & 14 & 9 & 2 & 2 \\
\hline FDA DailyMed & 16,968 & 1,754 & 743 & 215 & 119 & 76 & 32 & 23 & 10 & 6 & 16 & 2 & 2 & 1 \\
\hline $\begin{array}{l}\text { PDB ligand } \\
\text { Combiphos }\end{array}$ & 4,229 & 708 & 145 & 72 & 40 & 23 & 18 & 6 & 11 & 3 & - & 3 & - & - \\
\hline Catalyst & 1,900 & 141 & 33 & 4 & - & 1 & - & - & - & - & - & - & - & - \\
\hline MolMall-MDPI & 5,100 & 374 & 262 & 76 & 17 & 6 & 3 & 2 & - & 1 & 1 & - & - & - \\
\hline $\begin{array}{l}\text { Cayman } \\
\text { DrugBank }\end{array}$ & 2,687 & 789 & 100 & 61 & 15 & 12 & 8 & 3 & 2 & 4 & 1 & 3 & 3 & 1 \\
\hline$(5.1 .4)$ & 7,566 & 657 & 281 & 90 & 29 & 18 & 8 & 9 & 8 & 2 & 4 & 2 & - & - \\
\hline DrugCentral & 2,325 & 253 & 50 & 21 & 7 & 5 & - & 2 & 1 & 1 & 2 & 1 & - & 1 \\
\hline
\end{tabular}




\section{$\underline{\text { Transforms Associated with Conflicts }}$}

Table 4 shows the counts of transforms we were able to associate with intra-database conflicts identified based on E_ISOTOPE_HASH128 and E_ISOTOPE_TAUTO_HASH128. We had to use non-stereo sensitive hashcodes for this analysis because the explicit handling of stereogenic elements in the STEREO hashcodes prevents the straightforward generation of tautomeric pathways (see above), i.e. one tautomer may not converge to another tautomer. A total of 73 out of the 119 tested rules yielded at least one case of tautomeric conflict, be it a single- or multiplerule conflict. We note that the vast majority of conflicts are based on one of the common rules as mentioned above, possibly in combination with one or a few other rules, these being in the majority common rules themselves. We do however find some conflicts based on rare rules, including more than 1,000 cases involving new rules going beyond the 20 standard CACTVS rules: 435 single-rule conflicts, 1,277 multiple-rule conflicts..

Table 4. Counts of associated rules in single-rule conflicts as well as rule combinations associated with conflicts

$\begin{array}{lrr}\text { Rule number } & \text { Single-rule conflicts } & \text { Combined or/and alternative rule conflicts }^{\mathbf{b}} \\ \text { PT_02_00 } & 1,337 & 31,868 \\ \text { PT_03_00 } & 316 & 68,591 \\ \text { PT_04_00 } & - & 15,889 \\ \text { PT__00 } & 6 & 122,429 \\ \text { PT_05_00 } & 190,015 & 271,174 \\ \text { PT_06_00 } & 40,094 & 43,028 \\ \text { PT_07_00 } & 4 & 25,528 \\ \text { PT_08_00 } & 6,124 & 59,634 \\ \text { PT_09_00 } & 98 & 4,807 \\ \text { PT_10_00 } & 57 & 7,670 \\ \text { PT_11_00 } & 6 & 346 \\ \text { PT_11_01 } & 5 & 369 \\ \text { PT_11_02 } & 0 & 81 \\ \text { PT_11_03 } & 0 & 35 \\ \text { PT_11_04 } & - & 13,199 \\ \text { PT_12_00 } & 443 & 51 \\ \text { PT_13_00 } & - & 387 \\ \text { PT_14_00 } & 1 & 387 \\ \text { PT_15_00 } & 989 & 10,297 \\ \text { PT_16_00 } & - & 87 \\ \text { PT_17_00 } & 113 & 49 \\ \text { PT_18_00 } & 64 & 20 \\ \text { PT_19_00 } & 26 & 7 \\ \text { PT_20_00 } & 1,389 & 61 \\ \text { PT_21_00 } & 60 & 383 \\ \text { PT_22_00 } & & \end{array}$




\begin{tabular}{|c|c|c|}
\hline PT_23_00 & 254 & 42 \\
\hline PT_24_00 & - & 4 \\
\hline PT_27_00 & 58 & 40 \\
\hline PT_28_00 & - & 79 \\
\hline PT_29_00 & - & 58 \\
\hline PT_29_01 & 3 & 248 \\
\hline PT_32_00 & - & 42 \\
\hline PT_33_00 & - & 45 \\
\hline PT_35_00 & - & 2 \\
\hline PT_36_00 & - & 2 \\
\hline PT_37_00 & 6 & 7 \\
\hline PT_39_00 & 2 & 3 \\
\hline PT_41_00 & - & 4 \\
\hline PT_42_00 & 17 & 206 \\
\hline PT_44_00 & - & 6 \\
\hline PT_45_00 & 35 & 102 \\
\hline PT_47_00 & - & 4 \\
\hline RC_01_00 & - & 4 \\
\hline RC_02_00 & - & 1 \\
\hline RC_03_00 & - & 26 \\
\hline RC_03_01 & - & 19 \\
\hline RC_03_02 & - & 4 \\
\hline RC_04_00 & - & 9 \\
\hline RC_04_01 & - & 10 \\
\hline RC_04_02 & - & 13 \\
\hline RC_04_03 & - & 7 \\
\hline RC_05_00 & - & 3 \\
\hline RC_05_01 & - & 4 \\
\hline RC_05_02 & - & 3 \\
\hline RC_05_03 & - & 3 \\
\hline RC_06_00 & - & 10 \\
\hline RC_06_01 & - & 9 \\
\hline RC_06_02 & - & 3 \\
\hline RC_07_00 & - & 4 \\
\hline RC_07_01 & - & 4 \\
\hline RC_07_02 & - & 6 \\
\hline RC_07_03 & - & 55 \\
\hline RC_09_00 & - & 44 \\
\hline RC_09_01 & - & 23 \\
\hline RC_10_00 & - & 22 \\
\hline
\end{tabular}


RC_10_01

RC_10_02

RC_11_00

RC_11_01

RC_11_02

RC_20_00

VT_02_00

VT_06_00

$\begin{array}{ll}- & 22\end{array}$

$\begin{array}{ll}- & 13\end{array}$

- 2

$\begin{array}{ll}- & 2\end{array}$

- 2

- 1

$\begin{array}{ll}- & 3\end{array}$

$--1$

${ }^{a}$ Examining only single-rule transformations without any alternative rule possibility. ${ }^{b}$ Examining transformations involving alternative rules and/or multistep transformations.

We note that the rule combinations are mostly involving prototropic transforms, rarely ring-chain transforms and valence transforms.

\section{$\underline{\text { Percentage of conflicts involving non-persistent stereocenters }}$}

We have previously shown ${ }^{3}$ that tautomerism can change the stereochemistry of a compound: The changing location of a double bond may add or eliminate the presence of an E/Z stereo bond. Likewise, migration of a double bond to an $\mathrm{sp}^{3}$ hybridized atom that was chiral removes this chirality, which a further tautomeric isomerization step can re-establish with the opposite chirality, effectively creating racemization of this stereo center. Similarly, ring-chain equilibrium at an $\mathrm{sp}^{2}$ center can lead to generation of enantiomers, which was recently observed in two investigational molecules, where two ring tautomers of opposite chirality were in equilibrium with the chain form for each compound. ${ }^{9}$

The analysis of this effect in the context of this study was less straightforward than one may think. The issue is that tautomeric structures of the same molecule typically have different atom numbers, which makes atom-atom mapping challenging to determine whether a specific stereogenic center was affected by tautomerism or not. We instead compared the tautomeric overlaps based on stereo-sensitive hashcode identifiers with those based on stereo-invariant identifiers (called a "stereo tauto conflict" below). This entails the risk that structures with stereocenters that had different $\mathrm{R} / \mathrm{S}$ or $\mathrm{E} / \mathrm{Z}$ geometry, but did not participate in the tautomeric interconversion, and had no tautomerism-affected stereo centers, are erroneously counted. In detail, we extracted the subset of stereo tauto conflicts out of the overall conflicts in the following way: If the E_ISOTOPE_STEREO_HASH128 values were different from each other, and different from E_ISOTOPE_HASH128, and that one was the same as the E_ISOTOPE_STEREO_TAUTO_HASH128 value, then we designated this a case of stereo tauto conflict (being aware that there is a small possibility of mis-designation as outlined above) 
Table 5. Percentage of conflicts involving non-persistent stereocenters

Database

PubChem

eMolecules

SureChEMBL

(Patents)

MCULE

Ambinter

AMS

Screening

(2019 Q4)

MolPort

Enamine

InterChim

AsisChem

ChEMBL

Princeton

Biomolecular

Research

Life

Chemicals

ChemDiv

Vitas-M

Laboratory

Chemspace

ChemBridge

Innovapharm

TimTec

Alinda

US EPA

UORSY

Asinex

InterBioScreen

Otava

CSD Organic

HTS

Biochemie

ChemBank

Key Organic

ChemBlock

Maybridge

HMDB
Stereo conflict rate( $\%)$

67.47

27.01

38.66

22.04

8.54

43.56

26.22

50.80

59.26

44.17

78.30

12.01

0.92

9.77

15.73

39.95

92.01

49.46

22.34

55.12

21.89

0.00

85.57

21.09

12.50

89.16

0.31

7.52

59.55

16.55

12.96

31.34 
ChEBI

FDA

DailyMed

PDB ligand

Combiphos

Catalyst

MolMall-

MDPI

Cayman

DrugBank

(5.1.4)

DrugCentral

$40 \mathrm{DBs}$

merged
78.34

97.12

83.23

0.00

69.35

91.80

62.79

100.00

43.14

Table 5 shows a large spread of the percentages of conflicts involving non-persistent stereocenters, ranging from 0 to $100 \%$. This suggests that different databases treat stereochemistry differently, as well as that they differ in the types of compounds they contain that are affected by this effect. The average of the percentages of conflicts involving nonpersistent stereocenters was $42.6 \%$, and $43.1 \%$ when all databases were merged into one. We therefore see that this "destruction" of stereo centers by tautomerism is a common effect. The table with the absolute numbers of conflicts for each database is provided as Supporting Information Table S3.

\section{DISCUSSION}

The fact that the overall intra-database tautomeric conflict rate has changed little of the course of 10 years $^{3}$ indicates that not much appears to have changed in the recent past with how tautomerism is treated by database managers and providers.

We need to re-emphasize that identifying a tautomer conflict in a database does not per se indicate a problem with that database. There may be numerous reasons why several tautomers of the same molecule may be present in the same database. This includes the possibility that different tautomers were truly present in different samples, i.e. that the applied chemoinformatics rule was too "aggressive"; or that - especially in experimental databases - different conditions of measurement ${ }^{10}$ or sample preparation (e.g. in X-ray crystallography) yielded different tautomeric states.

Aggregating databases such as PubChem, combining data sets from hundreds of different sources ${ }^{11}$ are particularly prone to tautomeric conflicts. Strong normalization of input structures 
can reduce this effect but is fraught with some risks, including original submitters complaining, "What did you do to our structures?" We have previously analyzed a large database of commercially available samples ${ }^{5}$ and will therefore not further discuss this class of databases (labeled as " $\mathrm{S}$ " in Table 1).

As examples of our analyses, we present a handful of tautomeric conflicts we found for databases in the classes of regulatory agency databases ("R"), experimental results databases ("E”), and drug databases ("D”).

\section{US EPA}

The Distributed Structure-Searchable Toxicity (DSSTox) database of the US Environmental Protection Agency's (EPA) is a chemical database for predictive toxicology. Each chemical is mapped with bioassay and physicochemical property and toxicity data. The database preparation was described by the authors as being based on uniquely mapped identifiers (i.e., CAS RN, name and structure) and with rejection of entries that have any two identical identifiers. ${ }^{12}$ We found more than 2,777 tautomeric conflicts (see Supplementary Information Table S2) among the around 848,000 entries of this database. A few examples are shown in Figure 1.

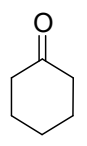

DTXCID00359<smiles>OC1=CCCCC1</smiles>

DTXCID10299584

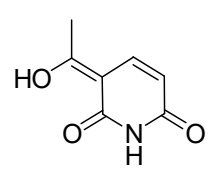

DTXCID10667123

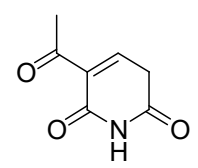

DTXCID30616613

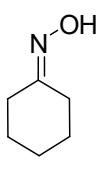

DTXCID701842

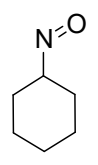

DTXCID70209124<smiles>Oc1ccc(N=Nc2ccccc2)cc1</smiles><smiles>O=C1C=CC(=NNc2ccccc2)C=C1</smiles>

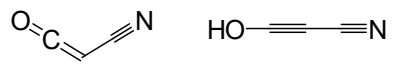

DTXCID10718886

DTXCID50118700

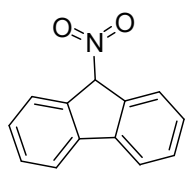

DTXCID401361281<smiles></smiles>

DTXCID50281660

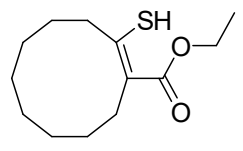

DTXCID00705922

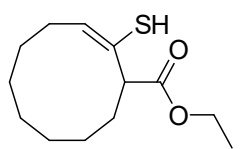

DTXCID60705923

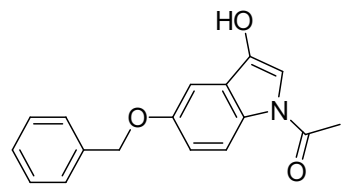

DTXCID00713683

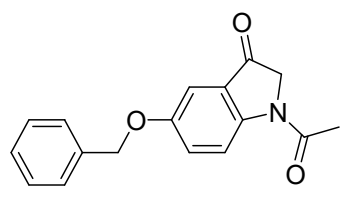

DTXCID80502456

Figure 1. Tautomeric conflict examples from EPA DSSTox. 


\section{FDA DailyMed}

FDA has been posting Substance Index SPL files for small-molecule based substances and biological substances (therapeutic proteins and biological organisms). From SPL files downloaded from the DailyMed website ${ }^{13}$, around 61,000 small molecules were collected from those SPL files that contained only a single small molecule. In these files each molecule has its own Unique Ingredient Identifier (UNII). ${ }^{14}$ Note that these structures are not limited to approved drugs but contain a wide variety of substances of interest to the FDA. We found a total of 556 tautomeric conflicts in this structure set. Figure 2 shows a few examples of tautomeric conflicts of structures having different UNIIs. We note, however, that we did not find any tautomeric conflicts amongactive ingredients in approved drugs (except for possible interchange of stereocenters upon tautomeric interconversion, see below). The conflicts we found involved other substances, which may indicate that registration of drug ingredients may undergo more comprehensive scrutiny than other substances of interest to FDA.

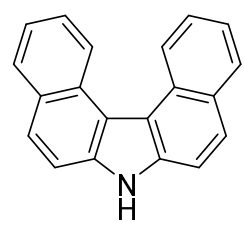

SZF10JI89D

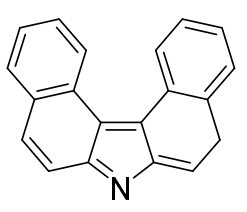

9SHH23M02S

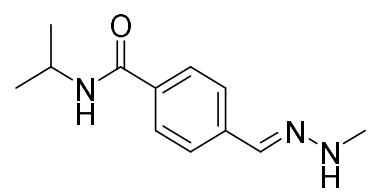

XF9EO27MN5

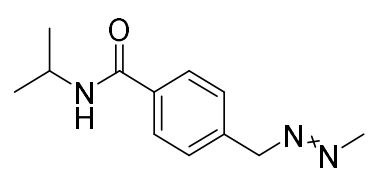

56768KS32D<smiles>CC(O)C(=O)c1nc2c(=O)nc(N)[nH]c2[nH]1</smiles>

CJQ26K07HP

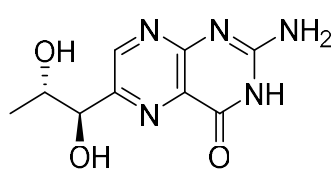

E54CTM794Y

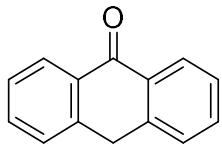

FPOFJ7K744

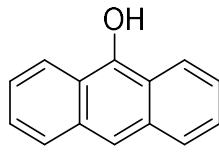

15PF96Z0OC

Figure 2. Tautomeric conflict examples from FDA Substance Indexing SPL files downloaded from DailyMed, with UNII identifiers shown.

\section{CSD Organic}

For the tautomeric analysis of the CSD, the collection of small molecule structures solved by Xray crystallography and neutron diffraction methods, we limited ourselves to only its organic molecules. From around 319,000 organic molecules, we found 452 cases where molecules exist in one or more alternative forms. In other databases, we term such pairs "tautomeric conflicts." However, in the case of the CSD they may be better called "alternative forms" in the same database because these are experimentally solved structures in which for the most part the 
hydrogens are resolved. Figure 3 shows some of the examples involving 1,3, 1,5, and 1,7 Hshifts, respectively.

In the WUQCOA/WEMRUD pair, the H-shift occurs between an $\mathrm{N}$ atom and an otherwise uncommonly seen Te atom. Other pairs involve hydrogen migration through aromatic phenyl and pyridine ring system (POFWOX/NEDMUF01 and AHPAZP10/CALWUA01).
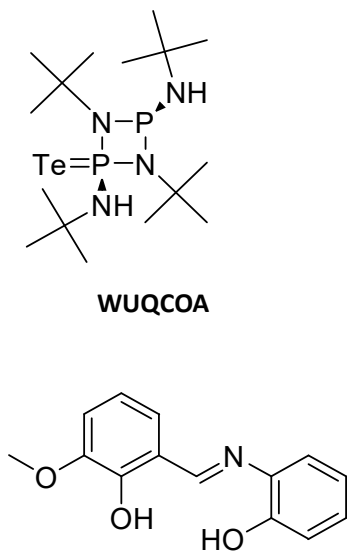

POFWOX

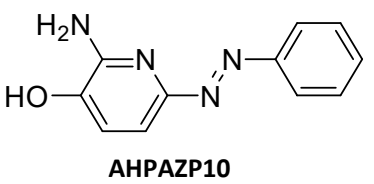

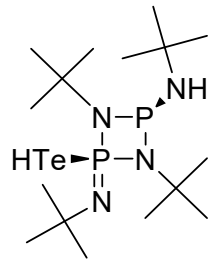

WEMRUD

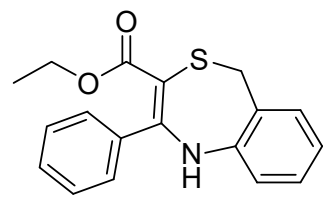

DUQMUY<smiles>CCOC(=O)[C@H]1SCc2ccccc2N=C1c1ccccc1</smiles>

DUQMOS<smiles>Cc1cc(=O)n2ncnc2[nH]1</smiles>

NESFIA<smiles>Cc1cc(O)n2ncnc2n1</smiles>

NESFIA01

Figure 3. Alternative pair examples from CSD.

\section{PDB Ligand Expo}

The PDB Ligand Expo database showed 167 tautomeric conflicts out of about 29,000 small molecule components reported in PDB entries bound to various macromolecules (protein, DNA). These different molecules are called "Unique Ligands" by the PDB. We could not find mentioning of a the possibility of presence of tautomeric molecules within this ligand set.

For example, from a set of four tautomers, three tautomers $(50 \mathrm{M}, 53 \mathrm{M}$ and $53 \mathrm{~L})$ are reported to be present in 5CDO (http://www.rcsb.org/structure/5CDO) and 1 tautomer (54Q) exists in 5CDM (http://www.rcsb.org/structure/5CDM) (Figure 4). One point to mention here is that all of these four tautomers have same standard InChI, thus InChI-based resolution of this case of tautomerism would not have been possible.

It is usually difficult to determine the tautomeric state of a bound molecule. Pyridoxamine-5phosphate-hydroxyisoxazole is an experimental molecule that is reported in three different 
tautomeric forms: 7TS, LCS and PMH in one (5U3F), four (6QP1, 4OMA, 1D7U and 4D9E) and two PDB entries (1XQK and $1 \mathrm{XQL})$, respectively.

For some molecules, one tautomer is reported to have high preference compared to another one. For example, the keto form of uric acid is reported in $22 \mathrm{PDB}$ entries whereas its hydroxy form occurs in only two PDB entries.<smiles>CC1CC2N(C1)c1ccc([N+](=O)[O-])cc1CC21C(=O)NC(=O)N=C1C(O)O</smiles>

$50 \mathrm{M}$

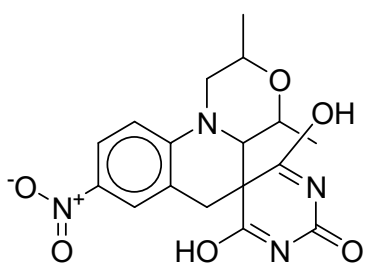

$53 \mathrm{M}$

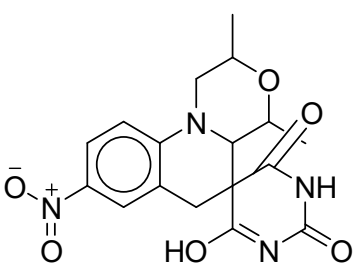

$53 \mathrm{~L}$<smiles>CC1CN2c3ccc([N+](=O)[O-])cc3CC3(C(=O)NC(=O)NC3=O)C2C(=O)O1</smiles>

54Q<smiles>Cc1ncc(COP(=O)(O)O)c(CNc2co[nH]c2=O)c1O</smiles>

7TS<smiles>Cc1ncc(COP(=O)(O)O)c(CN=C2CONC2=O)c1O</smiles>

LCS<smiles>Cc1ncc(COP(=O)(O)O)c(CNc2conc2O)c1O</smiles>

PMH

Figure 4. Tautomeric conflict examples among PDB ligands.

\section{DrugBank}

This database contains information on drugs and their targets. The main entries are from approved small molecule drugs, approved biologics (proteins, peptides, vaccines, and allergenics), nutraceuticals and experimental (discovery-phase) drugs. The major portion of DrugBank's information is related to chemical data and rest to the drugs' targets. ${ }^{15}$

DB00717 (Norethisterone) and DB09371 (Norethynodrel) are approved progesterone-based drugs. They are tautomers of each other based on our rules. (They also have different UNIIs: T18F433X4S and 88181ACA0M, respectively.) In other cases, one experimental drug shows conflict with other approved or/and experimental drug. DB00266 (Dicoumarol) and DB04392 (only the IUPAC name given in DrugBank) are an approved and an experimental drug, respectively, which form a tautomeric conflict. Similarly, an approved drug (DB00348, Nitisinone) is in tautomeric conflict with an experimental drug (DB08307). We note that some of 
the conflicts are based on stereocenters that are not tautomerism-persistent according to our rules. ${ }^{3}$<smiles>C=C[C@]1(O)CC[C@H]2[C@@H]3CCC4=CC(=O)CC[C@@H]4[C@H]3CC[C@]21C</smiles>

DB00717<smiles>O=c1oc2ccccc2c(O)c1Cc1c(O)c2ccccc2oc1=O</smiles>

DB00266<smiles>C=C[C@]1(O)CC[C@H]2C3CCC4=C(CCC(=O)C4)C3CC[C@@H]21</smiles>

DB09371<smiles>O=C1Oc2ccccc2C(=O)[C@H]1C[C@H]1C(=O)Oc2ccccc2C1=O</smiles>

DB04392<smiles>O=C1CCCC(=O)C1C(=O)c1ccc(C(F)(F)F)cc1[N+](=O)[O-]</smiles>

DB00348<smiles>Cc1ncc(COP(=O)(O)O)c(CNc2conc2O)c1O</smiles>

DB03097<smiles>O=C1CCCC(=O)C1=C(O)c1ccc(C(F)(F)F)cc1[N+](=O)[O-]</smiles>

DB08307<smiles>Cc1ncc(COP(=O)(O)O)c(CN=C2CON=C2O)c1O</smiles>

DB03787

Figure 5. Tautomeric conflict examples among DrugBank molecules.

\section{CONCLUSION}

Tautomeric conflicts are still found in virtually any database we look at. More comprehensive treatment of tautomerism therefore remains a desirable advance in chemistry, especially in database management and compound registration systems.

\section{SUPPORTING INFORMATION}

Table S1 contains all 119 transforms used in this paper, shown in SMIRKS format. It is an extension of a rule set we published previously. ${ }^{4}$ Table S2 shows the tautomeric conflict counts within each database for all tuple sizes, up to 25 . Table S3 shows the conflicts involving nonpersistent stereocenters ("stereo conflicts") for all tuple sizes, up to 25.

\section{AUTHOR INFORMATION}

\section{Corresponding Author}


* Computer-Aided Drug Design Group, Chemical Biology Laboratory, Center for Cancer

Research, National Cancer Institute, NIH, Frederick, MD 21702, USA. E-mail:

mn1@mail.nih.gov.

\section{Current Affiliation}

Devendra K Dhaked: Department of Pharmacoinformatics, National Institute of Pharmaceutical

Education and Research (NIPER), Kolkata - 700054 India

\section{ORCID}

Devendra K. Dhaked: 0000-0001-6349-7649

Marc C. Nicklaus: 0000-0002-4775-7030

\section{Competing interests}

The authors declare that they have no competing interests.

\section{Funding Sources}

The authors received funding from the NCI, NIH, Intramural Research Program.

\section{REFERENCES}

(1) Cruz-Cabeza, A. J.; Groom, C. R. Identification, Classification and Relative Stability of Tautomers in the Cambridge Structural Database. CrystEngComm 2011, 13 (1), 93-98. https://doi.org/10.1039/C0CE00123F.

(2) Milletti, F.; Storchi, L.; Sforna, G.; Cross, S.; Cruciani, G. Tautomer Enumeration and Stability Prediction for Virtual Screening on Large Chemical Databases. J. Chem. Inf. Model. 2009, 49 (1), 68-75. https://doi.org/10.1021/ci800340j.

(3) Sitzmann, M.; Ihlenfeldt, W.-D.; Nicklaus, M. C. Tautomerism in Large Databases. J. Comput. Aided Mol. Des. 2010, 24 (6-7), 521-551. https://doi.org/10.1007/s10822-0109346-4.

(4) Dhaked, D. K.; Ihlenfeldt, W.-D.; Patel, H.; Delannée, V.; Nicklaus, M. C. Toward a Comprehensive Treatment of Tautomerism in Chemoinformatics Including in InChI V2. $J$. Chem. Inf. Model. 2020, 60 (3), 1253-1275. https://doi.org/10.1021/acs.jcim.9b01080.

(5) Guasch, L.; Yapamudiyansel, W.; Peach, M. L.; Kelley, J. A.; Barchi, J. J.; Nicklaus, M. C. Experimental and Chemoinformatics Study of Tautomerism in a Database of Commercially Available Screening Samples. J. Chem. Inf. Model. 2016, 56 (11), 2149-2161. https://doi.org/10.1021/acs.jcim.6b00338. 
(6) Clemon, P. Private Communication. 2020.

(7) Ihlenfeldt, W. D.; Takahashi, Y.; Abe, H.; Sasaki, S. Computation and Management of Chemical Properties in CACTVS: An Extensible Networked Approach toward Modularity and Compatibility. J. Chem. Inf. Model. 1994, 34 (1), 109-116. https://doi.org/10.1021/ci00017a013.

(8) Guasch, L.; Sitzmann, M.; Nicklaus, M. C. Enumeration of Ring-Chain Tautomers Based on SMIRKS Rules. J. Chem. Inf. Model. 2014, 54 (9), 2423-2432. https://doi.org/10.1021/ci500363p.

(9) Ottosson, J. E.; Gränfors, M.; van Pelt, S.; Langborg Weinmann, A.; Nilsson Lill, S. O.; Hulthe, G.; Grönberg, G. Characterization and Demonstration of Drug Compound RingChain Tautomer Formation and Its Impacts on Quality Control. J. Pharm. Biomed. Anal. 2021, 198, 114020. https://doi.org/10.1016/j.jpba.2021.114020.

(10) Dhaked, D. K.; Guasch, L.; Nicklaus, M. C. Tautomer Database: A Comprehensive Resource for Tautomerism Analyses. J. Chem. Inf. Model. 2020, 60 (3), 1090-1100. https://doi.org/10.1021/acs.jcim.9b01156.

(11) PubChem Data Sources https://pubchem.ncbi.nlm.nih.gov/sources/ (accessed 2020 -04 -09).

(12) Grulke, C. M.; Williams, A. J.; Thillanadarajah, I.; Richard, A. M. EPA's DSSTox Database: History of Development of a Curated Chemistry Resource Supporting Computational Toxicology Research. Comput. Toxicol. 2019, 12, 100096. https://doi.org/10.1016/j.comtox.2019.100096.

(13) FDA DailyMed ftp://public.nlm.nih.gov/nlmdata/.dailymed/substance_indexing_spl_files.zip.

(14) FDA's Global Substance Registration System https://www.fda.gov/industry/fda-resourcesdata-standards/fdas-global-substance-registration-system (accessed 2020 -05-18).

(15) Wishart, D. S.; Feunang, Y. D.; Guo, A. C.; Lo, E. J.; Marcu, A.; Grant, J. R.; Sajed, T.; Johnson, D.; Li, C.; Sayeeda, Z.; Assempour, N.; Iynkkaran, I.; Liu, Y.; Maciejewski, A.; Gale, N.; Wilson, A.; Chin, L.; Cummings, R.; Le, D.; Pon, A.; Knox, C.; Wilson, M. DrugBank 5.0: A Major Update to the DrugBank Database for 2018. Nucleic Acids Res. 2018, 46 (D1), D1074-D1082. https://doi.org/10.1093/nar/gkx1037. 